\title{
Définir la valeur d'un point de vue sociologique
}

Define Value from a Sociological Point of View

\section{Nathalie Heinich}

\section{OpenEdition}

Journals

Édition électronique

URL : http://journals.openedition.org/recherchestravaux/1554

DOI : 10.4000/recherchestravaux.1554

ISSN : 1969-6434

\section{Éditeur}

UGA Éditions/Université Grenoble Alpes

\section{Édition imprimée}

ISBN : 978-2-37747-098-3

ISSN : 0151-1874

\section{Référence électronique}

Nathalie Heinich, «Définir la valeur d'un point de vue sociologique », Recherches \& Travaux [En ligne] 94 | 2019, mis en ligne le 20 juin 2019, consulté le 08 septembre 2020. URL : http://

journals.openedition.org/recherchestravaux/1554; DOI : https://doi.org/10.4000/recherchestravaux. 1554

Ce document a été généré automatiquement le 8 septembre 2020.

(c) Recherches \& Travaux 


\title{
Définir la valeur d'un point de vue sociologique
}

\author{
Define Value from a Sociological Point of View
}

\author{
Nathalie Heinich
}

1 Qu'est-ce qu'une valeur en littérature ? Ce peut être, premièrement, la valeur d'une œuvre («que vaut $L a$ Recherche du temps perdu?»), voire la valeur de «la » littérature en général : autrement dit, la valeur au sens de grandeur, de ce qui justifie qu'on s'intéresse à elle. Ce peut être aussi, deuxièmement, l'œuvre en tant qu'elle constitue une « valeur » dans le patrimoine culturel, autrement dit l'objet d'une valorisation à la fois collective, consensuelle et durable ( $L a$ Recherche du temps perdu comme "valeur » incontestée de la littérature française): autrement dit, la valeur au sens d'objet valorisé. Et ce peut être encore, troisièmement, le principe au nom duquel cette valorisation est accordée, conférant de «la" valeur, au premier sens, à un objet devenu «une " valeur, au deuxième sens: par exemple, la valeur de beauté, ou d'authenticité, ou de significativité, ou d'originalité, ou de pérennité, ou d'universalité : autrement dit, la valeur au sens de principe de valorisation.

2 Valeur-grandeur, valeur-objet, valeur-principe: à travers ces trois sens $d u$ mot «valeur ", l'on perçoit la complexité de cette problématique de la ou des valeurs - une problématique qu'il vaut la peine d'analyser avant de l'utiliser naïvement. C'est ce que je me propose de faire en résumant, à propos de l'objet «littérature», les principaux points de mon livre Des valeurs ${ }^{1}$. Je terminerai par quelques propositions susceptibles de guider plus concrètement la réflexion.

\section{En finir avec quelques idées reçues sur les valeurs}

3 Je commencerai par quelques idées reçues sur les valeurs, dont il serait bon de se débarrasser.

4 Une première idée reçue est que la notion de valeur se limiterait à ce que "vaut " une chose - la « valeur-grandeur », au premier sens. Ce serait oublier les biens auxquels on 
confère une valeur - les "valeurs-objets ", au deuxième sens -, que ces biens soient concrets - des choses, tel le manuscrit de La Recherche du temps perdu conservé dans une bibliothèque - ou abstraits - par exemple ce bien qu'est « la littérature ». Et ce serait oublier aussi - troisième sens - les «valeurs-principes", qui permettent de conférer de « la » valeur au premier sens, et qui se conjuguent forcément au pluriel, car il existe dans toute culture une pluralité de "valeurs ", au sens de principes organisant l'évaluation. C'est dire que «la » valeur, au premier sens, s'inscrit dans une pluralité d'objets « de » valeur, et résulte d'une pluralité de « valeurs » mobilisées pour en juger. Ainsi, en amont de la question de "la» valeur au singulier, qui tend à focaliser les débats, il importe de remonter "aux" valeurs qui la fondent, en faisant primer le pluriel « des » valeurs sur le singulier de « la » valeur.

5 Cette ouverture de la question axiologique - «axiologique » désignant ce qui a trait aux valeurs - à trois dimensions, et non plus à une seule, permet de démonter une deuxième idée reçue : celle selon laquelle il irait de soi de se focaliser sur le «juste prix » des choses. D'une part en effet, nous venons de le voir, ce premier sens du mot «valeur » n'est pas le seul; et d'autre part, même si l'on ne s'intéresse qu'à cette dimension de la "valeur-grandeur", celle-ci n'est pas réductible à son évaluation chiffrée qu'est le prix. Car celui-ci n'est que l'une des trois formes d'attribution de valeur, par la mesure chiffrée: or la valorisation peut se manifester aussi par l'attachement, et par le jugement. Mesure manifestée par les chiffres, attachement manifesté par les attitudes, jugement manifesté par les mots : voilà les trois grandes formes d'attribution de valeur, dont seule la première s'exprime essentiellement par le prix - et encore, uniquement lorsque l'évaluation porte sur des choses. Or les choses ne sont que l'une des quatre catégories d'objets susceptibles de donner lieu à évaluation: n'oublions pas que les personnes, les actions, et les états du monde (par exemple, l'état de la culture française) peuvent aussi être évalués. Toutefois, ni les personnes, ni les actions, ni les états du monde ne sont réductibles à une évaluation chiffrée, autrement dit à un prix.

6 C'est dire que la réduction de la problématique de la valeur au prix, autrement dit la monopolisation du terme de "valeur » par l'économie, opère une occultation massive des autres formes de valorisation - par l'attachement et par le jugement de valeur ainsi que des objets de valorisation qui ne sont pas des choses - autrement dit les personnes, les actions, les états du monde. En d'autres termes, la réduction de la valeur au prix revient à prendre l'arbre pour la forêt. Il suffit pour s'en convaincre d'imaginer comment nous réagirions si quelqu'un s'avisait de n'envisager La Recherche du temps perdu que sous l'angle de son prix...

7 Dès lors qu'on a accepté d'étendre la notion de valeur au-delà de la mesure, d'une part, et au-delà des choses, d'autre part, en s'intéressant aussi aux objets valorisés et aux principes de valorisation, une troisième idée reçue consiste à considérer que ces « valeurs » au pluriel - biens ou principes - sont particulièrement en phase avec une position droitière en politique. Certes, le vocabulaire des "valeurs" a été fortement sollicité par les partis de droite dans la France de la dernière génération, avec la focalisation sur la famille, la nation, la religion, l'autorité etc. ; mais l'acception progressiste, quoique moins facilement abritée derrière le mot de "valeur ", est tout aussi présente, s'agissant par exemple de mettre en exergue l'égalité, les droits de l'homme ou - de façon plus complexe aujourd'hui - la laïcité. Il est d'ailleurs facile de remarquer que des valeurs identifiées initialement à la sensibilité progressiste ont été 
peu à peu investies par la sensibilité conservatrice : ce fut le cas de la nation, du mérite, du travail et, plus récemment, de la laïcité. De même, certaines valeurs clivent à l'intérieur des camps politiques : aujourd'hui, la valeur républicaine de l'intérêt général se trouve aussi bien à droite qu'à gauche, de même que la valeur libérale de la liberté individuelle.

8 Autant dire que les valeurs peuvent changer de camp selon les contextes : pour la plupart, elles ne sont constitutivement ni de droite, ni de gauche. Et il en va de même, bien sûr, des valeurs mobilisées en littérature: Sade a longtemps été un auteur catalogué « de droite », conformément à l'éthique aristocratique du libertinage, avant de passer "à gauche " dans le courant du $\mathrm{xx}^{\mathrm{e}}$ siècle, au nom de la transgression des valeurs bourgeoises. Quant à Proust, ne cherchons même pas de quelle façon il a pu être revendiqué par des admirateurs de droite ou de gauche : ce serait considérer comme acquise la pertinence d'une lecture essentiellement politique des valeurs, alors même qu'une telle lecture est, à l'évidence, lourdement réductrice.

9 Une quatrième idée reçue, très répandue par la sociologie critique, héritière de la pensée matérialiste, consiste à réduire les valeurs à des intérêts cachés: elles ne seraient rien d'autre qu'une «idéologie » utilisée par les «dominants » pour asseoir leurs privilèges sur des principes qu'ils s'efforceraient de faire partager aux « dominés ${ }^{2}$ ». Or il suffit d'observer les jugements de valeur effectifs tels qu'ils s'expriment concrètement dans leurs contextes pour vérifier à quel point cette politisation simpliste de la question des valeurs est éloignée de la réalité. Et d'ailleurs, quel serait l'intérêt de classe dissimulé derrière la valeur accordée à La Recherche du temps perdu : la bourgeoisie chercherait-elle ainsi à imposer sa domination "symbolique " au peuple des exploités? L'aristocratie déchue se vengerait-elle en suscitant la fascination? Une telle lecture s'inscrirait dans la droite ligne de la réduction stalinienne de la culture et de la science à des enjeux politiques et sociaux : réduction qui a fait suffisamment la preuve, désormais, de son peu d'attention à la réalité du monde, et à sa complexité, pour qu'on ne lui accorde plus la moindre crédibilités.

Une cinquième idée reçue consiste à attribuer aux valeurs un caractère "sacré ", soit pour leur conférer du poids, soit, au contraire, pour les discréditer, en arguant de leur relativité et donc de leur absence de nécessité, propre à les " désacraliser ${ }^{4}$ ». Mais c'est là supposer que toute transcendance serait forcément religieuse ou, en d'autres termes, que « la religion » aurait le monopole de la transcendance, alors qu'elle n'en est qu'une des formes parmi d'autres: une erreur de raisonnement typique d'une pensée encore modelée dans le moule religieux. Que La Recherche du temps perdu possède aujourd'hui un statut à part dans la littérature française et même mondiale, qu'il fasse l'objet d'admirations voire de vénérations intenses, peut parfaitement s'analyser par l'intensité et la multiplicité des valorisations dont il fait l'objet, par la force des attachements éprouvés et manifestés par ses lecteurs, par la quantité d'analyses produites par les plus grands lettrés, voire par le prix qu'atteindrait son manuscrit autographe s'il était mis en vente. $Y$ accoler le qualificatif de « sacré » n'ajouterait rien à la compréhension, et même, probablement, la bloquerait, en nous persuadant que nous serions arrivés ainsi au bout de l'analyse. Pour comprendre un objet quelque peu mystérieux, tel que les valeurs, mieux vaut éviter de le réduire à un objet encore moins élucidé, tel que «le sacré ». Le paradigme religieux est sans pertinence concernant la question des valeurs et - comme souvent - arrête la pensée beaucoup plus qu'elle ne l'ouvre. 
11 Une sixième idée reçue consiste à réduire les valeurs à leurs ancrages contextuels, donc à les invalider par défaut d'universalité : ainsi, La Recherche du temps perdu ne serait une valeur « que » dans la société occidentale actuelle, par exemple, et ne pourrait donc pas légitimement prétendre au titre de "valeur» (au sens d'objet de valeur), parce que sa «valeur» (au sens de grandeur) ne serait pas reconnue ailleurs, et parce que les "valeurs » (au sens de principe) qui lui sont associées ne le seraient pas forcément dans d'autres cultures. Or toutes les valeurs sont contextuelles - comme le sont, d'ailleurs, toutes les formes de l'expérience humaine. Mais cela n'empêche nullement de les invoquer en tant que visées devant être partagées dans la société à laquelle on appartient. Qu'une représentation ne soit ni une substance ni un fait objectif existant en dehors de l'esprit humain, qu'elle soit donc "socialement construite ", voire "mythique ", ne lui ôte rien de son effectivité et de son efficacité, parce que la vie commune est faite non seulement de choses matérielles et d'institutions, mais aussi de conceptions plus ou moins partagées, qu'elles s'expriment par des mots, par des chiffres ou par des actes. Parmi ces représentations, les représentations «axiologiques", autrement dit les valeurs, ne sont pas les moins répandues ni les moins agissantes. En résumé, la relativité effective des valeurs n'empêche nullement qu'elles soient considérées comme des objectifs universels.

Une septième idée reçue consiste à réduire les valeurs à des «faits » objectifs, soit pour les valider, soit pour les invalider en en pointant la relativité ou la subjectivité, comme nous venons de le voir. Or l'on ne peut disqualifier une valeur en démontrant qu'elle "n'existe pas », au sens où elle ne serait pas effectivement mise en œuvre (par exemple, « l'égalité, ça n'existe pas ») : ce serait confondre une visée de l'action avec ses résultats. Ainsi, s'acharner à prouver que l'égalité dans le monde scolaire est une illusion parce que l'Éducation nationale ne parvient pas à assurer l'égalité des positions ni même des chances, c'est constater, certes, une réalité hautement critiquable, mais c'est en même temps s'aveugler sur ce qui permettrait de la faire progresser, en passant à côté de ce fait, fondamental en démocratie, qu'est le partage de la valeur d'égalité, en tant que mission impartie à l'institution scolaire - même si celle-ci la remplit mal. Et c'est donc, du même coup, miner en la disqualifiant la seule ressource permettant d'exiger la bonne mise en œuvre de cette mission. Ainsi, c'est au nom d'une conception de la démocratie vidée de toute prise au sérieux de la notion de valeurs, que certains, croyant œuvrer pour la démocratisation, s'ingénient à lui couper les ailes : c'est dire que les plus démystificateurs ne sont pas les moins mystifiés qui soient.

Enfin, une huitième et dernière idée reçue consiste à réduire les valeurs aux seules valeurs d'ordre éthique et politique. L'un des responsables de ce réductionnisme est la philosophie politique, qui tend à réduire les valeurs à la question de l'égalité, de la liberté, de la tolérance, de la démocratie, etc. ${ }^{5}$ : certes, ce sont des questions fondamentales, mais il serait dommage de s'y limiter lorsqu'on se propose d'évaluer une œuvre, ou de comprendre les principes qui président à son évaluation. L'autre responsable de ce réductionnisme est la philosophie morale, qui assimile spontanément toute valeur à la seule dimension morale ou éthique ${ }^{6}$ : même si celle-ci est fondamentale dans notre société, même si le registre éthique se caractérise par une très grande polysémie des valeurs concernées, et même si, dans une controverse, le passage par l'éthique est très souvent un gage de victoire des tenants de cette position, ce registre n'est pertinent que dans certains contextes, à propos de certains objets. L'exemple de La Recherche du temps perdu vous aura fait sentir à quel point, parfois, 
l'éthique est une dimension peu pertinente, voire déplacée dans notre système axiologique: savoir si le narrateur a pu se montrer cruel, si l'homosexualité est blâmable, si le snobisme est risible, sont des préoccupations qui ont leur place dans la vie ordinaire - celle qui constitue le cadre de nos interactions réelles - mais qui sont aujourd'hui considérées comme beaucoup trop «hétéronomes" pour s'appliquer utilement à la question de la valeur d'une œuvre littéraire.

\section{Définir la valeur-grandeur}

Ayant ainsi tenté de déminer un certain nombre de malentendus qui pourraient entraver la bonne compréhension de ce qu'est une valeur, je propose à présent d'appliquer, schématiquement, ces trois définitions - la valeur-grandeur, la valeurobjet, la valeur-principe - à un objet littéraire, pour observer ce que l'approche sociologique peut nous apporter. Je ne prendrai pas cet objet au hasard, bien sûr : j'en reste à mon exemple favori - celui de La Recherche du temps perdu...

Je définis la valeur, au premier sens du terme, comme « la résultante de l'ensemble des opérations par lesquelles une qualité est affectée à un objet ». Cette définition permet une approche "pragmatique ", c'est-à-dire centrée sur les opérations d'évaluation en situation réelle : opérations qui sont fonction à la fois de la nature de l'objet évalué, de la nature des sujets évaluateurs, et de la nature du contexte d'évaluation. Ce point est essentiel: l'on ne peut pas comprendre ni même observer un processus d'évaluation sans prendre en compte ces trois paramètres que sont l'objet, le sujet, le contexte.

L'objet, à savoir l'œuvre littéraire, est au centre des préoccupations des spécialistes de littérature, qui disposent d'une vaste batterie d'outils pour analyser les propriétés de - par exemple - La Recherche du temps perdu. À cet intérêt pour l'objet évalué, la sociologie, sous sa forme explicative, ajoute un intérêt pour les sujets évaluateurs : comment la valeur accordée à cette œuvre varie selon les catégories sociales, les classes d'âge, les sexes, les niveaux d'études, voire les générations. Mais il existe une autre sociologie, dont on est peut-être moins familier, car elle n'emprunte pas aux méthodes quantitatives des sondages d'opinion mais aux méthodes qualitatives pratiquées par les ethnologues; cette sociologie-là - dite analytique ou compréhensive - nous amène à diriger notre regard vers cette réalité éminemment sociologique, mais insuffisamment prise en compte, qu'est le contexte d'évaluation. Celui-ci peut s'appréhender soit à un niveau très général - par exemple, le contexte de la société française actuelle, avec le bagage culturel qui lui est propre -, soit à un niveau très particulier - par exemple, une situation de débat dans une salle de classe à propos de l'œuvre de Proust, ou bien un colloque universitaire où des spécialistes exposent leurs points de vue, ou encore une confrontation entre experts lors d'un litige au tribunal à propos de l'authenticité d'un manuscrit présumé autographe.

Cette approche «pragmatique » des valeurs, à travers l'étude empirique des processus d'évaluation, est la seule à même de rendre compte de leur complexité, et de produire une analyse sociologique qui soit tout à la fois neutre, descriptive, empirique et compréhensive. Il faut prendre en compte toutes ces dimensions - le contexte « micro » et «macro » de l'évaluation, l'identité des sujets évaluateurs, les propriétés des objets évalués - pour rendre compte du processus par lequel un objet se voit doté de "valeur ", devenant ainsi lui-même une "valeur ", grâce à la mise en œuvre d'un certain nombre de "valeurs ». C'est dire qu'à l'image des opérations qui la produisent, 
la valeur n'est ni objective, ni subjective, ni arbitraire : elle est motivée par les prises qu'offre l'objet à l'évaluation, par les représentations collectives dont sont dotés les individus, et par les possibilités qu'offrent les contextes d'activation de ces représentations à propos d'un objet.

\section{Décrire la valeur-objet}

Intéressons-nous à présent à ce qui fait de La Recherche du temps perdu une "valeur » au deuxième sens du terme, c'est-à-dire un bien. Comment cette œuvre en est-elle venue à devenir un fleuron de la littérature?

Pour répondre à cette question, il faudrait re-parcourir les différents contextes dans lesquels, à propos de cet objet particulier, différentes catégories d'acteurs ont produit des évaluations susceptibles d'être acceptées comme des valorisations. Nous voilà dans le programme de l'histoire littéraire et, plus précisément, l'histoire sociale de la réception. Rôle des éditeurs, reconnaissance par les pairs, intérêt des critiques littéraires, puis des spécialistes de littérature voire de philosophie, engouement de lecteurs de plus en plus nombreux et diversifiés, réputation grandissante au-delà de la France, traductions, introduction dans les manuels scolaires, publication de biographies de l'auteur: telles sont les grandes étapes de la production d'un « chefd'œuvre » de la littérature, à travers la série des « cercles de reconnaissance » qu'il doit traverser, des intermédiaires les plus spécialisés au grand public.

Ces étapes ont déjà été décrites, du moins en partie, par les spécialistes d'histoire littéraire et de sociologie de la réception ${ }^{7}$. C'est là une dimension désormais bien connue de la recherche universitaire, dédiée à la question - même si elle n'est pas toujours identifiée comme telle - de savoir comment une œuvre devient une « valeur » littéraire.

\section{Analyser les valeurs-principes}

21 Reste la question qui m'intéresse le plus, car elle a été le moins étudiée : c'est celle qui occupe toute la troisième partie de mon livre Des Valeurs. Une approche sociologique ${ }^{8}$. Il s'agit d'analyser la façon dont un objet - ici, une œuvre littéraire - se voit doté de «valeur » au premier sens, et devient donc une «valeur» au deuxième sens, par des jugements de valeur faisant intervenir des «valeurs » au troisième sens, c'est-à-dire des principes d'évaluation.

Dans le cas de La Recherche du temps perdu, il s'agirait de repérer quels sont ces principes selon qu'ils interviennent dans tel ou tel contexte d'évaluation (en famille, entre amis, à l'école, dans une thèse universitaire, dans un manuel scolaire...), et selon qu'ils sont énoncés par telle ou telle catégorie d'acteurs (simples lecteurs, élèves, chercheurs, enseignants...). Je n'ai pas fait le travail, mais il n'est pas difficile - s'agissant de notre propre culture - d'imaginer quels ils peuvent être : la valeur de beauté, appliquée au style ou à l'œuvre entière; la valeur de virtuosité, appliquée à la maîtrise du style et de la construction narrative ; la valeur d'authenticité, appliquée à l'accès qu'elle nous livre à l'intériorité de l'auteur, ou à nos propres émotions profondes; la valeur de plaisir, appliquée à ce que nous éprouvons à la lire ; la valeur de significativité, appliquée aux dimensions cachées que cette œuvre recèle; la valeur de spiritualité, appliquée à des 
entités transcendantes à laquelle elle nous donnerait accès - la condition humaine, la création, le temps...; la valeur affective, appliquée aux sentiments portés par les personnages; ou encore les valeurs d'originalité, de pérennité et d'universalité reconnues à l'œuvre.

Les choses pourraient toutefois se compliquer pour peu que l'on prenne en compte non plus les valorisations mais aussi les dévalorisations, qu'il faut neutraliser pour permettre à l'œuvre d'accéder au statut de bien incontestable, doté d'une grandeur universellement reconnue. Or les détracteurs ne manquent pas, ou n'ont pas manqué : illisibilité (registre technique de l'efficacité), laideur des phrases trop longues (registre esthétique de la beauté), immoralité des sujets abordés (registre éthique de la moralité), difficulté de venir à bout d'un récit trop long et trop complexe (registre æsthésique du plaisir) - etc.

Notons que j'introduis ici un deuxième niveau d'analyse: au-dessus des valeurs, les «registres de valeurs", autrement dit les familles regroupant des valeurs apparentées - par exemple, pour le registre éthique, la moralité, la décence, le respect des religions, la sensibilité à la souffrance, la charité, l'empathie, etc. Ce niveau des registres de valeurs est particulièrement utile pour se repérer dans les controverses, et en comprendre la logique. En outre, les choses deviendraient plus complexes encore si l'on introduisait - en-dessous et non plus au-dessus du niveau des valeurs - le niveau des critères, autrement dit des nombreuses caractéristiques permettant d'attribuer une valeur - par exemple, pour la beauté, le critère de l'élégance stylistique, ou de la construction narrative, ou de la richesse sémantique, etc. Enfin, sous le niveau des critères, il faudrait aussi analyser les "prises » qu'offre l'objet à l'application de ces critères, autorisant eux-mêmes la mise en œuvre de valeurs appartenant à tel ou tel registre de valeur. Ces "prises" sont les nombreuses propriétés objectives qui permettent aux évaluateurs de s'emparer perceptivement de l'objet et, éventuellement, de l'évaluer: par exemple, la longueur des phrases, l'usage de tel ou tel mot, les digressions narratives, etc.

Prises, critères, valeurs, registres de valeurs : ainsi se présente, sous forme pyramidale, la «grammaire axiologique » telle que j'ai tenté de la mettre en évidence à travers l'étude des évaluations effectives. Elle comporte également, au-dessus des registres de valeurs, les quatre «amplificateurs de valeurs » (l'originalité, la pérennité, la rareté, l'universalité), au statut un peu particulier dans la mesure où il s'agit de valeurs potentiellement présentes dans tous les registres, où elles amplifient les autres valeurs; ainsi enfin que deux grands "régimes de qualification" (régime de communauté, régime de singularité), qui déterminent la valeur positive ou négative accordée à ce qui est commun et à ce qui est hors du commun, et que j'ai identifiés dans mes précédents travaux en sociologie de l'art et de la littérature ${ }^{9}$. Cette modélisation du système d'évaluation permet d'y voir plus clair dans maintes controverses, aussi bien que dans les processus qui permettent de les clore.

Il n'existe plus guère aujourd'hui de contestation audible de la «valeur » qu'il convient d'accorder à La Recherche du temps perdu, devenu une «valeur " littéraire de premier plan grâce à sa capacité à accueillir la mise en œuvre de multiples « valeurs » qui nous sont familières. Mais derrière cette familiarité se cachent des processus complexes et passionnants, qui éclairent tant la genèse de notre système de valeurs que ses éventuelles mises en cause. C'est cette «boîte noire » de l'évaluation que j'ai souhaité ouvrir, et dont j'espère avoir fait sentir l'intérêt. 


\section{La transmission des valeurs}

pu éclairer sur ce que peut apporter l'approche sociologique en matière d'analyse, de compréhension, de définition de ce que sont les valeurs, mais guère sur l'enseignement des valeurs et, plus précisément, leur enseignement par la littérature. Rien n'empêche en effet d'utiliser une œuvre littéraire pour sensibiliser des élèves à la question des valeurs, et leur faire sentir leur importance. Même si cela peut paraître inadéquat à l'autonomie du texte littéraire aux yeux des spécialistes, cet usage pédagogique des œuvres me paraît non seulement possible - il n'enlève rien à l'œuvre - et souhaitable - il apporte quelque chose à ses lecteurs. Je souhaiterais donc terminer par quelques propositions plus ciblées sur cette question.

Si j'ai utilisé le terme de "grammaire axiologique », c'est que la comparaison avec la linguistique m'a paru pertinente et éclairante. Ainsi, de même que le grammairien décrit un système mais ne prescrit pas une norme, le sociologue décrit un système axiologique mais n'a pas à prescrire de normes d'évaluation : la neutralité demeure un impératif catégorique du travail du chercheur, même et surtout s'agissant d'objets aussi chargés de normativité que les valeurs. En ce sens le sociologue et le professeur de français œuvrent de façons très différentes, voire opposées, puisque l'un se doit d'éviter toute normativité, tandis que l'autre est, au contraire, payé pour enseigner des normes linguistiques et familiariser les élèves avec les œuvres situées au sommet de la hiérarchie des valeurs littéraires. Mais rien n'empêche de considérer que le travail descriptif du premier puisse être utile au travail normatif du second, de même que la description du grammairien aide l'enseignant à formaliser la transmission des normes.

Par ailleurs, l'on peut considérer que, comme la compétence linguistique, la compétence axiologique est à la fois, de fait, plus ou moins développée selon les acteurs, et, en droit, considérée comme universelle, du moins dans l'espace linguistique concerné. Nous partageons tous le même système de valeurs, même si nous ne le mettons pas forcément en œuvre de la même façon selon les contextes et les objets. La relativité effective, associée à l'universalité de principe, est une propriété constitutive de notre rapport aux valeurs, comme elle l'est de notre rapport à la langue.

Un autre bénéfice de l'analogie linguistique réside dans la dimension pédagogique. $\mathrm{Si}$ les œuvres peuvent avoir un usage pédagogique, en tant qu'elles sont porteuses de valeurs - notamment les valeurs éthiques et civiques -, c'est que la "compétence axiologique " - la capacité à mettre en œuvre des évaluations susceptibles d'être adaptées à leurs objets et à leurs contextes - n'est pas une compétence innée, mais acquise. C'est la position que je défends, car la compétence axiologique me paraît être du même ordre que la compétence linguistique : un système que nous apprenons par l'imprégnation, autrement dit par l'acculturation, et qui peut être éventuellement complété par un enseignement systématique, comme c'est le cas avec l'apprentissage de la langue à l'école. Or, de même que l'on apprend à parler en écoutant ses proches, puis en recevant un enseignement spécifique, de même il n'est pas absurde de penser que l'on apprend à évaluer non seulement en écoutant la façon dont nos proches évaluent, mais aussi en recevant une formation aux valeurs. C'est, d'ailleurs, l'objectif de l'éducation morale et civique. Rien n'empêche de considérer que celle-ci peut aussi passer par l'analyse des textes littéraires. 

de personnages imaginaires ou de personnes réelles. La description de leurs émotions nous donne accès aux valeurs dont la transgression suscite de telles émotions - car les émotions, et notamment l'indignation, sont souvent des indicateurs de valeurs à travers l'expérience de leur transgression ${ }^{10}$. La description des sentiments active l'empathie et, à travers elle, l'expérience de l'injustice ou de la méchanceté - mais aussi, ne l'oublions pas, l'expérience de l'atteinte à nos critères de beauté, de sincérité, de sens du jeu, de plaisir, etc.

En ce sens, l'explicitation des valeurs qui sous-tendent les récits littéraires peut être un excellent exercice pédagogique, à condition toutefois de pouvoir les déployer dans toutes leurs dimensions. Ainsi, il est possible de mettre en évidence, selon les cas, la façon dont un texte est susceptible de donner prise à des évaluations relevant du registre esthétique (valeurs de beauté, d'élégance stylistique...), du registre æsthésique (valeurs de plaisir, d'excitation...), du registre éthique (valeurs de décence, de sensibilité à la souffrance, d'empathie, de religiosité, de charité, de dédication à autrui...), du registre civique (valeurs de responsabilité, d'engagement...), du registre pur (valeurs d'authenticité, de sincérité...), du registre herméneutique (valeurs de sens, de significativité...), du registre mystique (valeurs de transcendance, d'inspiration...), $\mathrm{du}$ registre ludique (valeurs de jeu, d'humour, d'ironie...), du registre domestique (valeurs de solidarité familiale, de respect des anciens...), du registre affectif (valeurs d'amour, d'attachement...), du registre réputationnel (valeurs d'honneur, de renommée...), du registre technique (valeurs d'efficacité, de virtuosité...), du registre fonctionnel (valeurs d'utilité, de réparation...), et même du registre économique (valeurs de richesse, de profit...). L'on peut également relever les modalités d'amplification de ces valeurs par ces « valeurs cardinales » que sont l'ancienneté et la nouveauté, l'universalité et la rareté, ainsi que leur inscription dans le «régime de communauté » ou le « régime de singularité ». L'on peut enfin pointer les éventuelles tensions entre ces valeurs hétérogènes, susceptibles d'engendrer des conflits de registres de valeurs, sources de controverses durables.

C'est cette ouverture du spectre axiologique, à travers une explicitation du système de valeurs que nous partageons tous plus ou moins, que j'ai proposée dans mon livre. Il reste à espérer qu'elle pourra avoir aussi une utilité pratique pour les enseignants.

\section{NOTES}

1. Voir N. Heinich, Des valeurs. Une approche sociologique, Paris, Gallimard, 2017.

2. Voir notamment P. Bourdieu, La Domination masculine, Paris, Seuil, 1998 ; P. Bourdieu, H. Haacke, Libre échange, Paris, Seuil / Les Presses du réel, 1994.

3. Pour une critique de ce réductionnisme, voir P. Bourdieu, « Le marché des biens symboliques », L'Année sociologique, vol. 22, 1973.

4. Pour un exemple récent de réduction au sacré, voir B. Lahire, Ceci n'est pas qu'un tableau, Paris, La Découverte, 2015.

Recherches \& Travaux, 94 | 2019 
5. Voir par exemple R. Inglehart, The Silent Revolution: Changing Values and Political Styles among Western Publics, Princeton, Princeton University Press, 1977.

6. Voir par exemple B. Williams, Ethics and the Limits of Philosophy, Harvard, Cambridge University Press, 1981.

7. Voir notamment H. R. Jauss, Pour une esthétique de la réception [1975], trad. Cl. Maillard, Paris, Gallimard, 1978.

8. N. Heinich, Des Valeurs. Une approche sociologique, Paris, Gallimard, coll. «Bibliothèque des sciences humaines ", 2017.

9. Voir N. Heinich, La Gloire de Van Gogh. Essai d'anthropologie de l'admiration, Paris, Minuit, 1991 ; L'Épreuve de la grandeur. Prix littéraires et reconnaissance, Paris, La Découverte, 1999 ; Être écrivain. Création et identité, Paris, La Découverte, 2000 ; L'Élite artiste. Excellence et singularité en régime démocratique, Paris, Gallimard, 2005 ; La Fabrique du patrimoine. De la cathédrale à la petite cuillère, Paris, éditions de la Maison des sciences de l'homme, 2009 ; Le paradigme de l'art contemporain. Structures d'une révolution artistique, Paris, Gallimard, 2012.

10. À ce sujet, voir notamment P. Livet, Émotions et rationalité morale, Paris, PUF, 2002.

\section{RÉSUMÉS}

Après avoir tenté de dissiper un certain nombre d'idées reçues concernant les valeurs, l'article s'attache à définir les trois sens du mot «valeur» tels que peut les appréhender la sociologie axiologique (valeur-grandeur, valeur-objet, valeur-principe), à travers des exemples pris à l'histoire littéraire. Il propose pour finir plusieurs pistes concernant la transmission des valeurs dans le cadre pédagogique, notamment à travers la notion de " grammaire axiologique».

After dismissing a number of missleading common places about values, the article offers a definition of the three meanings of the word "value" as evidenced by axiological sociology (value as worth, value as object, value as principle), through examples taken from literary history. Finally, it suggests several ways of transmissing values in the pedagogical framework, in particular through the notion of "axiological grammar".

\section{AUTEUR}

\section{NATHALIE HEINICH}

Directeur de recherches au CNRS, Nathalie Heinich est l'auteur de nombreux ouvrages, dont la plupart traitent directement ou indirectement de la question des valeurs, que ce soit dans l'art contemporain (Le Paradigme de l'art contemporain, 2014), le statut de l'artiste (L'Élite artiste, 2005 ; De la visibilité, 2012), la reconnaissance littéraire (L'Épreuve de la grandeur, 1999) ou le patrimoine (La Fabrique du patrimoine, 2009). 